\title{
The Casimir Effect: Some Aspects
}

\author{
Carlos Farina \\ Universidade Federal do Rio de Janeiro, Ilha do Fundão, \\ Caixa Postal 68528, Rio de Janeiro, RJ, 21941-972, Brazil
}

Received on 10 August, 2006

\begin{abstract}
We start this paper with a historical survey of the Casimir effect, showing that its origin is related to experiments on colloidal chemistry. We present two methods of computing Casimir forces, namely: the global method introduced by Casimir, based on the idea of zero-point energy of the quantum electromagnetic field, and a local one, which requires the computation of the energy-momentum stress tensor of the corresponding field. As explicit examples, we calculate the (standard) Casimir forces between two parallel and perfectly conducting plates and discuss the more involved problem of a scalar field submitted to Robin boundary conditions at two parallel plates. A few comments are made about recent experiments that undoubtedly confirm the existence of this effect. Finally, we briefly discuss a few topics which are either elaborations of the Casimir effect or topics that are related in some way to this effect as, for example, the influence of a magnetic field on the Casimir effect of charged fields, magnetic properties of a confined vacuum and radiation reaction forces on non-relativistic moving boundaries.
\end{abstract}

Keywords: Quantum field theory; Casimir effect

\section{INTRODUCTION}

\section{A. Some history}

The standard Casimir effect was proposed theoretically by the dutch physicist and humanist Hendrik Brugt Gerhard Casimir (1909-2000) in 1948 and consists, basically, in the attraction of two parallel and perfectly conducting plates located in vacuum [1]. As we shall see, this effect has its origin in colloidal chemistry and is directly related to the dispersive van der Waals interaction in the retarded regime.

The correct explanation for the non-retarded dispersive van der Walls interaction between two neutral but polarizable atoms was possible only after quantum mechanics was properly established. Using a perturbative approach, London showed in 1930 [2] for the first time that the above mentioned interaction is given by $V_{L o n}(r) \approx-(3 / 4)\left(\hbar \omega_{0} \alpha^{2}\right) / r^{6}$, where $\alpha$ is the static polarizability of the atom, $\omega_{0}$ is the dominant transition frequency and $r$ is the distance between the atoms. In the 40's, various experiments with the purpose of studying equilibrium in colloidal suspensions were made by Verwey and Overbeek [3]. Basically, two types of force used to be invoked to explain this equilibrium, namely: a repulsive electrostatic force between layers of charged particles adsorbed by the colloidal particles and the attractive London-van der Waals forces.

However, the experiments performed by these authors showed that London's interaction was not correct for large distances. Agreement between experimental data and theory was possible only if they assumed that the van der Waals interaction fell with the distance between two atoms more rapidly than $1 / r^{6}$. They even conjectured that the reason for such a different behaviour for large distances was due to the retardation effects of the electromagnetic interaction (the information of any change or fluctuation occurred in one atom should spend a finite time to reach the other one). Retardation effects must be taken into account whenever the time interval spent by a light signal to travel from one atom to the other is of the order (or greater) than atomic characteristic times $\left(r / c \geq 1 / \omega_{M a n}\right.$, where $\omega_{m n}$ are atomic transition frequencies). Though this conjecture seemed to be very plausible, a rigorous demonstration was in order. Further, the precise expression of the van der Waals interaction for large distances (retarded regime) should be obtained.

Motivated by the disagreement between experiments and theory described above, Casimir and Polder [4] considered for the first time, in 1948, the influence of retardation effects on the van der Waals forces between two atoms as well as on the force between an atom and a perfectly conducting wall. These authors obtained their results after lengthy calculations in the context of perturbative quantum electrodynamics (QED). Since Casimir and Polder's paper, retarded forces between atoms or molecules and walls of any kind are usually called Casimir-Polder forces. They showed that in the retarded regime the van der Waals interaction potential between two atoms is given by $V_{R e t}(r)=-23 \hbar c \alpha_{A} \alpha_{B} /\left(4 \pi r^{7}\right)$. In contrast to London's result, it falls as $1 / r^{7}$. The change in the power law of the dispersive van der Waals force when we go from the non-retarded regime to the retarded one $\left(F_{N R} \sim 1 / r^{7} \rightarrow F_{R} \sim 1 / r^{8}\right)$ was measured in an experiment with sheets of mica by D. Tabor and R.H.S. Winterton [5] only 20 years after Casimir and Polder's paper. A change was observed around $150 \AA$, which is the order of magnitude of the wavelength of the dominant transition (they worked in the range $50 \AA-300 \AA$, with an accuracy of $\pm 4 \AA$ ). They also showed that the retarded van der Waals interaction potential between an atom and a perfectly conducting wall falls as $1 / r^{4}$, in contrast to the result obtained in the short distance regime (non-retarded regime), which is proportional to $1 / r^{3}$ (as can be seen by the image method). Casimir and Polder were very impressed with the fact that after such a lengthy and involved QED calculation, the final results were extremely simple. This is very clear in a conversation with Niels Bohr. In Casimir's own words 
In the summer or autumn 1947 (but I am not absolutely certain that it was not somewhat earlier or later) I mentioned my results to Niels Bohr, during a walk. "That is nice", he said, "That is something new." I told him that I was puzzled by the extremely simple form of the expressions for the interaction at very large distance and he mumbled something about zero-point energy. That was all, but it put me on a new track.

Following Bohr's suggestion, Casimir re-derived the results obtained with Polder in a much simpler way, by computing the shift in the electromagnetic zero-point energy caused by the presence of the atoms and the walls. He presented his result in the Colloque sur la théorie de la liaison chimique, that took place at Paris in April of 1948:

Ifound that calculating changes of zero-point energy really leads to the same results as the calculations of Polder and myself...

A short paper containing this beautiful result was published in a French journal only one year later [6]. Casimir, then, decided to test his method, based on the variation of zero-point energy of the electromagnetic field caused by the interacting bodies in other examples. He knew that the existence of zeropoint energy of an atomic system (a hot stuff during the years that followed its introduction by Planck [7]) could be inferred by comparing energy levels of isotopes. But how to produce isotopes of the quantum vacuum? Again, in Casimir's own words we have the answer [8]:

\section{if there were two isotopes of empty space you could really easy confirm the existence of the zero-point energy. Unfortunately, or perhaps for- tunately, there is only one copy of empty space and if you cannot change the atomic distance then you might change the shape and that was the idea of the attracting plates.}

A month after the Colloque held at Paris, Casimir presented his seminal paper [1] on the attraction between two parallel conducting plates which gave rise to the famous effect that since then bears his name:

On 29 May, 1948, 'I presented my paper on the attraction between two perfectly conducting plates at a meeting of the Royal Netherlands Academy of Arts and Sciences. It was published in the course of the year..

As we shall see explicitly in the next section, Casimir obtained an attractive force between the plates whose modulus per unit area is given by

$$
\frac{F(a)}{L^{2}} \approx 0,013 \frac{1}{(a / \mu m)^{4}} \frac{d y n}{c m^{2}},
$$

where $a$ is the separation between the plates, $L^{2}$ the area of each plate (presumably very large, i.e., $L \gg a$ ).
A direct consequence of dispersive van der Waals forces between two atoms or molecules is that two neutral but polarizable macroscopic bodies may also interact with each other. However, due to the so called non-additivity of van der Waals forces, the total interaction potential between the two bodies is not simply given by a pairwise integration, except for the case where the bodies are made of a very rarefied medium. In principle, the Casimir method provides a way of obtaining this kind of interaction potential in the retarded regime (large distances) without the necessity of dealing explicitly with the non-additivity problem. Retarded van der Waals forces are usually called Casimir forces. A simple example may be in order. Consider two semi-infinite slabs made of polarizable material separated by a distance $a$, as shown in Figure 1 .

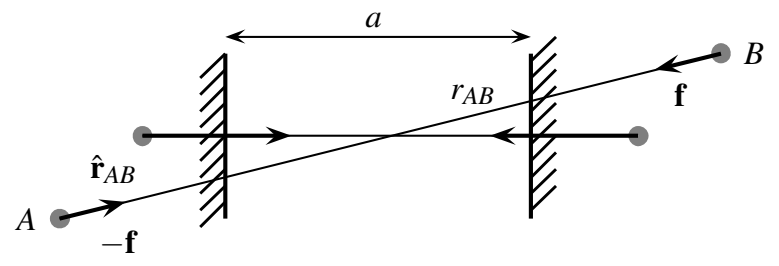

FIG. 1: Forces between molecules of the left slab and molecules of the right slab.

Suppose the force exerted by a molecule $A$ of the left slab on a molecule $B$ of the right slab is given by

$$
\mathbf{f}_{A B}=-\frac{C}{r_{A B}^{\gamma}} \mathbf{r}_{A B}
$$

where $C$ and $\gamma$ are positive constants, $r_{A B}$ the distance between the molecules and $\hat{\mathbf{r}}_{A B}$ the unit vector pointing from $A$ to $B$. Hence, by a direct integration it is straightforward to show that, for the case of dilute media, the force per unit area between the slabs is attractive and with modulus given by

$$
\frac{F_{\text {slabs }}}{\text { Area }}=\frac{C^{\prime}}{a^{\gamma-4}},
$$

where $C^{\prime}$ is a positive constant. Observe that for $\gamma=8$, which corresponds to the Casimir and Polder force, we obtain a force between the slabs per unit area which is proportional to $1 / a^{4}$. Had we used the Casimir method based on zero-point energy to compute this force we would have obtained precisely this kind of dependence. Of course, the numerical coefficients would be different, since here we made a pairwise integration, neglecting the non-additivity problem. A detailed discussion on the identification of the Casimir energy with the sum of van der Waals interaction for a dilute dielectric sphere can be found in Milton's book [9] (see also references therein).

In 1956, Lifshitz and collaborators developed a general theory of van der Waals forces [10]. They derived a powerful expression for the force at zero temperature as well as at finite temperature between two semi-infinite dispersive media characterized by well defined dielectric constants and separated by a slab of any other dispersive medium. They were 
able to derive and predict several results, like the variation of the thickness of thin superfluid helium films in a remarkable agreement with the experiments [11]. The Casimir result for metallic plates can be reobtained from Lifshitz formula in the appropriate limit. The Casimir and Polder force can also be inferred from this formula [9] if we consider one of the media sufficiently dilute such that the force between the slabs may be obtained by direct integration of a single atom-wall interaction [12].

The first experimental attempt to verify the existence of the Casimir effect for two parallel metallic plates was made by Sparnaay [13] only ten years after Casimir's theoretical prediction. However, due to a very poor accuracy achieved in this experiment, only compatibility between experimental data and theory was established. One of the great difficulties was to maintain a perfect parallelism between the plates. Four decades have passed, approximately, until new experiments were made directly with metals. In 1997, using a torsion pendulum Lamoreaux [14] inaugurated the new era of experiments concerning the Casimir effect. Avoiding the parallelism problem, he measured the Casimir force between a plate and a spherical lens within the proximity force approximation [15]. This experiment may be considered a landmark in the history of the Casimir effect, since it provided the first reliable experimental confirmation of this effect. One year later, using an atomic force microscope, Mohideen and Roy [16] measured the Casimir force between a plate and a sphere with a better accuracy and established an agreement between experimental data and theoretical predictions of less than a few percents (depending on the range of distances considered). The two precise experiments mentioned above have been followed by many others and an incomplete list of the modern series of experiments about the Casimir effect can be found in [17]-[26]. For a detailed analysis comparing theory and experiments see $[27,28]$

We finish this subsection emphasizing that Casimir's original predictions were made for an extremely idealized situation, namely: two perfectly conducting (flat) plates at zero temperature. Since the experimental accuracy achieved nowadays is very high, any attempt to compare theory and experimental data must take into account more realistic boundary conditions. The most relevant ones are those that consider the finite conductivity of real metals and roughness of the surfaces involved. These conditions become more important as the distance between the two bodies becomes smaller. Thermal effects must also be considered. However, in principle, these effects become dominant compared with the vacuum contribution for large distances, where the forces are already very small. A great number of papers have been written on these topics since the analysis of most recent experiments require the consideration of real boundary conditions. For finite conductivity effects see Ref. [30]; the simultaneous consideration of roughness and finite conductivity in the proximity for approximation can be found in Ref. [31] and beyond PFA in Ref. [32] (see also references cited in the above ones). Concerning the present status of controversies about the thermal Casimir force see Ref. [29]

\section{B. The Casimir's approach}

The novelty of Casimir's original paper was not the prediction of an attractive force between neutral objects, once London had already explained the existence of a force between neutral but polarizable atoms, but the method employed by Casimir, which was based on the zero-point energy ot the electromagnetic field. Proceeding with the canonical quantization of the electromagnetic field without sources in the Coulomb gauge we write the hamiltonian operator for the free radiation field as

$$
\hat{H}=\sum_{\alpha=1}^{2} \sum_{\mathbf{k}} \hbar \omega_{\mathbf{k}}\left[\hat{a}_{\mathbf{k} \alpha}^{\dagger} \hat{a}_{\mathbf{k} \alpha}+\frac{1}{2}\right]
$$

where $\hat{a}_{\mathbf{k} \alpha}^{\dagger}$ and $\hat{a}_{\mathbf{k} \alpha}$ are the creation and annihilation operators of a photon with momentum $\mathbf{k}$ and polarization $\alpha$. The energy of the field when it is in the vacuum state, or simply the vacuum energy, is then given by

$$
\mathcal{E}_{0}:=\langle 0|\hat{H}| 0\rangle=\sum_{\mathbf{k}} \sum_{\alpha=1}^{2} \frac{1}{2} \hbar \omega_{\mathbf{k}},
$$

which is also referred to as zero-point energy of the electromagnetic field in free space. Hence, we see that even if we do not have any real photon in a given mode, this mode will still contribute to the energy of the field with $\frac{1}{2} \hbar \omega_{\mathbf{k} \alpha}$ and total vacuum energy is then a divergent quantity given by an infinite sum over all possible modes.

The presence of two parallel and perfectly conducting plates imposes on the electromagnetic field the following boundary conditions:

$$
\begin{gathered}
\mathbf{E} \times\left.\hat{\mathbf{n}}\right|_{\text {plates }}=\mathbf{0} \\
\left.\mathbf{B} \cdot \hat{\mathbf{n}}\right|_{\text {plates }}=0,
\end{gathered}
$$

which modify the possible frequencies of the field modes. The Casimir energy is, then, defined as the difference between the vacuum energy with and without the material plates. However, since in both situations the vacuum energy is a divergent quantity, we need to adopt a regularization prescription to give a physical meaning to such a difference. Therefore, a precise definition for the Casimir energy is given by

$$
\mathcal{E}_{\text {Cas }}:=\lim _{s \rightarrow 0}\left[\left(\sum_{\mathbf{k} \alpha} \frac{1}{2} \hbar \omega_{\mathbf{k}}\right)_{I}-\left(\sum_{\mathbf{k} \alpha} \frac{1}{2} \hbar \omega_{\mathbf{k}}\right)_{I I}\right],
$$

where subscript $I$ means a regularized sum and that the frequencies are computed with the boundary conditions taken into account, subscript $I I$ means a regularized sum but with no boundary conditions at all and $s$ stands for the regularizing parameter. This definition is well suited for plane geometries like that analyzed by Casimir in his original work. In more complex situations, like those involving spherical shells, there are some subtleties that are beyond the purposes of this introductory article (the self-energy of a spherical shell depends on 
its radius while the self-energy of a pair of plates is independent of the distance between them).

Observe that, in the previous definition, we eliminate the regularization prescription only after the subtraction is made. Of course, there are many different regularization methods. A quite simple but very efficient one is achieved by introducing a high frequency cut off in the zero-point energy expression, as we shall see explicitly in the next section. This procedure can be physically justified if we note that the metallic plates become transparent in the high frequency limit so that the high frequency contributions are canceled out from equation (6).

Though the calculation of the Casimir pressure for the case of two parallel plates is very simple, its determination may become very involved for other geometries, as is the case, for instance, of a perfectly conducting spherical shell. After a couple of years of hard work and a "nightmare in Bessel functions", Boyer [33] computed for the first time the Casimir pressure inside a spherical shell. Surprisingly, he found a repulsive pressure, contrary to what Casimir had conjectured five years before when he proposed a very peculiar model for the stability of the electron [34]. Since then, Boyer's result has been confirmed and improved numerically by many authors, as for instance, by Davies in 1972 [35], by Balian and Duplantier in 1978 [36] and also Milton in 1978 [37], just to mention some old results.

The Casimir effect is not a peculiarity of the electromagnetic field. It can be shown that any relativistic field under boundary conditions caused by material bodies or by a compactification of space dimensions has its zero-point energy modified. Nowadays, we denominate by Casimir effect any change in the vacuum energy of a quantum field due to any external agent, from classical backgrounds and non-trivial topology to external fields or neighboring bodies. Detailed reviews of the Casimir effect can be found in [9, 38-41].

\section{A local approach}

In this section we present an alternative way of computing the Casimir energy density or directly the Casimir pressure which makes use of a local quantity, namely, the energymomentum tensor. Recall that in classical electromagnetism the total force on a distribution of charges and currents can be computed integrating the Maxwell stress tensor through an appropriate closed surface containing the distribution. For simplicity, let us illustrate the method in a scalar field. The lagrangian density for a free scalar field is given by

$$
\mathcal{L}\left(\phi, \partial_{\mu} \phi\right)=-\frac{1}{2} \partial_{\mu} \phi \partial^{\mu} \phi-\frac{1}{2} m^{2} \phi^{2}
$$

The field equation and the corresponding Green function are given, respectively, by

$$
\begin{aligned}
\left(-\partial^{2}+m^{2}\right) \phi(x) & =0 ; \\
\left(\partial^{2}-m^{2}\right) G\left(x, x^{\prime}\right) & =-\delta\left(x-x^{\prime}\right),
\end{aligned}
$$

where, as usual, $G\left(x, x^{\prime}\right)=i\left\langle 0\left|T\left(\phi(x) \phi\left(x^{\prime}\right)\right)\right| 0\right\rangle$.

Since the above lagrangian density does not depend explicitly on $x$, Noether's Theorem leads naturally to the following energy-momentum tensor $\left(\partial_{\mu} T^{\mu v}=0\right)$

$$
T^{\mu v}=\frac{\partial \mathcal{L}}{\partial\left(\partial_{\mu} \phi\right)} \partial^{v} \phi+g^{\mu v} \mathcal{L}
$$

which, after symmetrization, can be written in the form

$$
T_{\mu v}=\frac{1}{2}\left(\partial_{\mu} \phi \partial_{v} \phi+\partial_{v} \phi \partial_{\mu} \phi\right)+g_{\mu v} \mathcal{L}
$$

For our purposes, it is convenient to write the vacuum expectation value $(\mathrm{VEV})$ of the energy-momentum tensor in terms of the above Green function as

$$
\left\langle 0\left|T_{\mu v}(x)\right| 0\right\rangle=-\frac{i}{2} \lim _{x^{\prime} \rightarrow x}\left[\left(\partial_{\mu}^{\prime} \partial_{\nu}+\partial_{v}^{\prime} \partial_{\mu}\right)-g_{\mu v}\left(\partial_{\alpha}^{\prime} \partial^{\alpha}+m^{2}\right)\right] G\left(x, x^{\prime}\right) .
$$

In this context, the Casimir energy density is defined as

$$
\rho_{C}(x)=\left\langle 0\left|T_{00}(x)\right| 0\right\rangle_{B C}-\left\langle 0\left|T_{00}(x)\right| 0\right\rangle_{\text {Free }},
$$

where the subscript $B C$ means that the VEV must be computed assuming that the field satisfies the appropriate boundary condition. Analogously, considering for instance the case of two parallel plates perpendicular to the $O Z$ axis (the generalization for other configurations is straightforward) the Casimir force per unit area on one plate is given by

$$
\mathcal{F}_{C}=\left\langle 0\left|T_{z z}^{+}\right| 0\right\rangle-\left\langle 0\left|T_{z z}^{-}\right| 0\right\rangle,
$$

where superscripts + and - mean that we must evaluate $\left\langle T_{z z}\right\rangle$ on both sides of the plate. In other words, the desired Casimir pressure on the plate is given by the discontinuity of $\left\langle T_{z z}\right\rangle$ at the plate. Using equation (12), $\left\langle T_{z z}\right\rangle$ can be computed by

$$
\left\langle 0\left|T_{z z}\right| 0\right\rangle=-\frac{i}{2} \lim _{x^{\prime} \rightarrow x}\left(\frac{\partial}{\partial z} \frac{\partial}{\partial z^{\prime}}-\frac{\partial^{2}}{\partial z^{2}}\right) G\left(x, x^{\prime}\right) .
$$

Local methods are richer than global ones, since they provide much more information about the system. Depending on the problem we are interested in, they are indeed necessary, as for instance in the study of radiative properties of an atom inside a cavity. However, with the purpose of computing Casimir energies in simple situations, one may choose, for convenience, global methods. Previously, we presented only the global method introduced by Casimir, based on the zero-point energy of the quantized field, but there are many others, namely, the generalized zeta function method [42] and Schwinger's method [43, 44], to mention just a few.

\section{EXPLICIT COMPUTATION OF THE CASIMIR FORCE}

In this section, we show explicitly two ways of computing the Casimir force per unit area in simple situations where plane surfaces are involved. We start with the global approach 
introduced by Casimir which is based on the zero-point energy. Then, we give a second example where we use a local approach, based on the energy-momentum tensor. We finish this section by sketching some results concerning the Casimir effect for massive fields.

\section{A. The electromagnetic Casimir effect between two parallel plates}

As our first example, let us consider the standard (QED) Casimir effect where the quantized electromagnetic field is constrained by two perfectly parallel conducting plates separated by a distance $a$. For convenience, let us suppose that one plate is located at $z=0$, while the other is located at $z=a$. The quantum electromagnetic potential between the metallic plates in the Coulomb gauge $(\nabla \cdot \mathbf{A}=0)$ which satisfies the appropriate $\mathrm{BC}$ is given by [45]

$$
\begin{aligned}
\mathbf{A}(\rho, z, t) & =\frac{L^{2}}{(2 \pi)^{2}} \sum_{n=0}^{\infty} \int d^{2} \kappa\left(\frac{2 \pi \hbar}{c k a L^{2}}\right)^{1 / 2} \times \\
& \times\left\{a^{(1)}(\kappa, n)(\hat{\kappa} \times \hat{\mathbf{z}}) \sin \left(\frac{n \pi z}{a}\right)+\right. \\
& \left.+a^{(2)}(\kappa, n)\left[i \frac{n \pi}{k a} \kappa \sin \left(\frac{n \pi z}{a}\right)-\hat{\mathbf{z}} \frac{\kappa}{k} \cos \left(\frac{n \pi z}{a}\right)\right]\right\} \times \\
& \times e^{i(\kappa \cdot \rho-\omega t)}+\text { h.c. },
\end{aligned}
$$

where $\omega(\kappa, n)=c k=c\left[\kappa^{2}+n^{2} \pi^{2} / a^{2}\right]^{1 / 2}$, with $n$ being a non-negative integer and the prime in $\Sigma^{\prime}$ means that for $n=0$ an extra $1 / 2$ factor must be included in the normalization of the field modes. The non-regularized Casimir energy then reads

$$
\begin{aligned}
\mathcal{E}_{c}^{n r}(a)=\frac{\hbar c}{2} & \int L^{2} \frac{d^{2} \kappa}{(2 \pi)^{2}}\left[\kappa+2 \sum_{n=1}^{\infty}\left(\kappa^{2}+\frac{n^{2} \pi^{2}}{a^{2}}\right)^{1 / 2}\right] \\
& -\frac{\hbar c}{2} \int L^{2} \frac{d^{2} \kappa}{(2 \pi)^{2}} \int_{-\infty}^{+\infty} \frac{a d k_{z}}{2 \pi} 2 \sqrt{\kappa^{2}+k_{z}^{2}}
\end{aligned}
$$

Making the variable transformation $\kappa^{2}+(n \pi / a)^{2}=: \lambda$ and introducing exponential cutoffs we get a regularized expression (in 1948 Casimir used a generic cutoff function),

$$
\begin{aligned}
\mathcal{E}^{r}(a, \varepsilon)=\frac{L^{2}}{2 \pi} & {\left[\frac{1}{2} \int_{0}^{\infty} e^{-\varepsilon \kappa} \kappa^{2} d \kappa+\sum_{n=1}^{\infty} \int_{\frac{n \pi}{a}}^{\infty} e^{-\varepsilon \lambda} \lambda^{2} d \lambda-\right.} \\
& \left.-\int_{0}^{\infty} d n \int_{\frac{n \pi}{a}}^{\infty} e^{-\varepsilon \lambda} \lambda^{2} d \lambda\right] \\
=\frac{L^{2}}{2 \pi} & {\left[\frac{1}{\varepsilon^{3}}+\sum_{n=1}^{\infty} \frac{\partial^{2}}{\partial \varepsilon^{2}}\left(\frac{e^{-\varepsilon n \pi / a}}{\varepsilon}\right)-\right.} \\
& \left.-\int_{0}^{\infty} d n \frac{\partial^{2}}{\partial \varepsilon^{2}} \int_{\frac{n \pi}{a}}^{\infty} e^{-\varepsilon \lambda} d \lambda\right]
\end{aligned}
$$

Using that

$$
\frac{\partial^{2}}{\partial \varepsilon^{2}}\left[\frac{1}{\varepsilon} \sum_{n=1}^{\infty} e^{-\varepsilon n \pi / a}\right]=\frac{\partial^{2}}{\partial \varepsilon^{2}}\left[\frac{1}{\varepsilon} \frac{1}{e^{\varepsilon \pi / a}-1}\right]
$$

as well as the definition of Bernoulli's numbers,

$$
\frac{1}{e^{t}-1}=\sum_{n=0}^{\infty} B_{n} \frac{t^{n-1}}{n !}
$$

we obtain

$$
\begin{aligned}
\mathcal{E}(a) & =\frac{L^{2}}{2 \pi}\left[\frac{1}{\varepsilon^{3}}+\frac{\partial^{2}}{\partial \varepsilon^{2}}\left\{\frac{1}{\varepsilon} \sum_{n=0}^{\infty} \frac{B_{n}}{n !}\left(\frac{\varepsilon \pi}{a}\right)^{n-1}\right\}-\frac{6 a}{\pi \varepsilon^{4}}\right] \\
& =\frac{L^{2}}{2 \pi}\left[6\left(B_{0}-1\right) \frac{a}{\pi} \frac{1}{\varepsilon^{4}}+\left(1+2 B_{1}\right) \frac{1}{\varepsilon^{3}}+\frac{B_{4}}{12}\left(\frac{\pi}{a}\right)^{3}\right. \\
& \left.+\sum_{n=5}^{\infty} \frac{B_{n}}{n !}\left(\frac{\pi}{a}\right)^{n-1}(n-2)(n-3) \varepsilon^{n-4}\right] .
\end{aligned}
$$

Using the well known values $B_{0}=1, B_{1}=-\frac{1}{2}$ and $B_{4}=-\frac{1}{30}$, and taking $\varepsilon \rightarrow 0^{+}$, we obtain

$$
\frac{\mathcal{E}(a)}{L^{2}}=-\hbar c \frac{\pi^{2}}{24 \times 30} \cdot \frac{1}{a^{3}}
$$

As a consequence, the force per unit area acting on the plate at $z=a$ is given by

$$
\frac{F(a)}{L^{2}}=-\frac{1}{L^{2}} \frac{\partial \mathcal{E}_{c}(a)}{\partial a}=-\frac{\pi^{2} \hbar c}{240 a^{4}} \approx-0,013 \frac{1}{(a / \mu m)^{4}} \frac{d y n}{c m^{2}},
$$

where in the last step we substituted the numerical values of $\hbar$ and $c$ in order to give an idea of the strength of the Casimir pressure. Observe that the Casimir force between the (conducting) plates is always attractive. For plates with $1 \mathrm{~cm}^{2}$ of area separated by $1 \mu \mathrm{m}$ the modulus of this attractive force is $0,013 \mathrm{dyn}$. For this same separation, we have $P_{\text {Cas }} \approx 10^{-8} P_{\text {atm }}$, where $P_{\text {atm }}$ is the atmospheric pressure at sea level. Hence, for the idealized situation of two perfectly conducting plates and assuming $L^{2}=1 \mathrm{~cm}^{2}$, the modulus of the Casimir force would be $\approx 10^{-7} N$ for typical separations used in experiments. However, due to the finite conductivity of real metals, the Casimir forces measured in experiments are smaller than these values.

\section{B. The Casimir effect for a scalar field with Robin BC}

In order to illustrate the local method based on the energymomentum tensor, we shall discuss the Casimir effect of a massless scalar field submitted to Robin BC at two parallel plates, which are defined as

$$
\left.\phi\right|_{\text {bound. }}=\left.\beta \frac{\partial \phi}{\partial n}\right|_{\text {bound. }},
$$


where, by assumption, $\beta$ is a non-negative parameter. However, before computing the desired Casimir pressure, a few comments about Robin $\mathrm{BC}$ are in order.

First, we note that Robin BC interpolate continuously Dirichlet and Neumann ones. For $\beta \rightarrow 0$ we reobtain Dirichlet BC while for $\beta \rightarrow \infty$ we reobtain Neumann BC. Robin BC already appear in classical electromagnetism, classical mechanics, wave, heat and Schrödinger equations [46] and even in the study of interpolating partition functions [47]. A nice realization of these conditions in the context of classical mechanics can be obtained if we study a vibrating string with its extremes attached to elastic supports [48? ]. Robin BC can also be used in a phenomenological model for a penetrable surfaces [49]. In fact, in the context of the plasma model, it can be shown that for frequencies much smaller than the plasma frequency $\left(\omega \ll \omega_{P}\right)$ the parameter $\beta$ plays the role of the plasma wavelength [?]. In the context of QFT this kind of BC appeared more than two decades ago $[50,51]$. Recently, they have been discussed in a variety of contexts, as in the AdS/CFT correspondence [52], in the discussion of upper bounds for the ratio entropy/energy in confined systems [53], in the static Casimir effect [54], in the heat kernel expansion [55-57] and in the one-loop renormalization of the $\lambda \phi^{4}$ theory $[58,59]$. As we will see, Robin BC can give rise to restoring forces in the static Casimir effect [54].

Consider a massless scalar field submitted to Robin BC on two parallel plates:

$$
\left.\phi\right|_{z=0}=\left.\beta_{1} \frac{\partial \phi}{\partial z}\right|_{z=0} ;\left.\phi\right|_{z=a}=-\left.\beta_{2} \frac{\partial \phi}{\partial z}\right|_{z=a}
$$

where we assume $\beta_{1}\left(\beta_{2}\right) \geq 0$. For convenience, we write

$$
G\left(x, x^{\prime}\right)=\int \frac{d^{3} k_{\alpha}}{(2 \pi)^{3}} e^{\imath k_{\alpha}\left(x-x^{\prime}\right)^{\alpha}} g\left(z, z^{\prime} ; \mathbf{k}_{\perp}, \omega\right),
$$

with $\alpha=0,1,2, g_{\mu \nu}=\operatorname{diag}(-1,+1,+1,+1)$ and the reduced Green function $g\left(z, z^{\prime} ; \mathbf{k}_{\perp}, \omega\right)$ satisfies

$$
\left(\frac{\partial^{2}}{\partial z^{2}}+\lambda^{2}\right) g\left(z, z^{\prime} ; \mathbf{k}_{\perp}, \omega\right)=-\delta\left(z-z^{\prime}\right),
$$

where $\lambda^{2}=\omega^{2}-\mathbf{k}_{\perp}^{2}$ and $g\left(z, z^{\prime} ; \mathbf{k}_{\perp}, \omega\right)$ is submitted to the following boundary conditions (for simplicity, we shall not write $\mathbf{k}_{\perp}, \omega$ in the argument of $g$ ):

$$
g\left(0, z^{\prime}\right)=\beta_{1} \frac{\partial}{\partial z} g\left(0, z^{\prime}\right) ; \quad g\left(a, z^{\prime}\right)=-\beta_{2} \frac{\partial}{\partial z} g\left(a, z^{\prime}\right)
$$

It is not difficult to see that $g\left(z, z^{\prime}\right)$ can be written as

$$
g\left(z, z^{\prime}\right)= \begin{cases}A\left(z^{\prime}\right)\left(\sin \lambda z+\beta_{1} \lambda \cos \lambda z\right), & z<z^{\prime} \\ B\left(z^{\prime}\right)\left(\sin \lambda(z-a)-\beta_{2} \lambda \cos \lambda(z-a)\right), z>z^{\prime}\end{cases}
$$

For points inside the plates, $0<z, z^{\prime}<a$, the final expression for the reduced Green function is given by

$$
g^{R R}\left(z, z^{\prime}\right)=-\frac{\left(\frac{1}{\gamma_{1}} e^{\imath \lambda z_{<}}-e^{-\imath \lambda z_{<}}\right)\left(\frac{1}{\gamma_{2}} e^{\imath \lambda\left(z_{>}-a\right)}-e^{\imath \lambda\left(z_{>}-a\right)}\right)}{2 \imath \lambda\left(\frac{1}{\gamma_{1}} e^{\imath \lambda a}-\frac{1}{\gamma_{2}} e^{-\imath \lambda a}\right)},
$$

where $\gamma_{i}=\frac{1+i \beta_{i} \lambda}{1-i \beta_{i} \lambda}(i=1,2)$. Outside the plates, with $z, z^{\prime}>a$, we have:

$$
g^{R R}\left(z, z^{\prime}\right)=\frac{e^{\imath \lambda\left(z_{<}-a\right)}}{2 \imath \lambda}\left(\frac{1}{\gamma_{2}} e^{\imath \lambda\left(z_{<}-a\right)}-e^{-\imath \lambda\left(z_{<}-a\right)}\right) .
$$

Defining $t^{\mu v}$ such that

$$
\left\langle T^{\mu v}(x)\right\rangle=\int \frac{d^{2} \mathbf{k}_{\perp}}{(2 \pi)} \frac{d \omega}{2 \pi}\left\langle t^{\mu v}(x)\right\rangle
$$

we have

$$
\left\langle t^{33}(z)\right\rangle=\frac{1}{2 i} \lim _{z^{\prime} \rightarrow z}\left(\frac{\partial}{\partial z} \frac{\partial}{\partial z^{\prime}}+\lambda^{2}\right) g\left(z, z^{\prime}\right) .
$$

The Casimir force per unit area on the plate at $x=a$ is given by the discontinuity in $\left\langle t^{33}\right\rangle$ :

$$
\mathcal{F}=\int \frac{d^{2} \mathbf{k}_{\perp}}{(2 \pi)} \frac{d \omega}{2 \pi}\left[\left.\left\langle t^{33}\right\rangle\right|_{z=a_{-}}-\left.\left\langle t^{33}\right\rangle\right|_{z=a_{+}}\right] .
$$

After a straightforward calculation, it can be shown that

$$
\mathcal{F}\left(\beta_{1}, \beta_{2} ; a\right)=-\frac{1}{32 \pi^{2} a^{4}} \int_{0}^{\infty} \frac{d \xi^{3}}{\left(\frac{1+\beta_{1} \xi / 2 a}{1-\beta_{1} \xi / 2 a}\right)\left(\frac{1+\beta_{2} \xi / 2 a}{1-\beta_{2} \xi / 2 a}\right) e^{\xi}-1} .
$$

Depending on the values of parameters $\beta_{1}$ and $\beta_{2}$, restoring Casimir forces may arise, as shown in Figure 2 by the dotted line and the thin solid line.

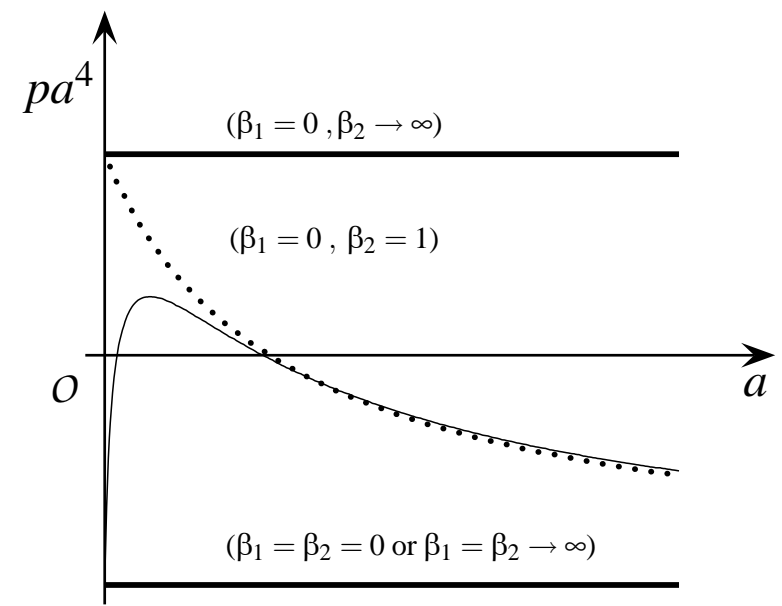

FIG. 2: Casimir pressure, conveniently multiplied by $a^{4}$, as a function of $a$ for various values of parameters $\beta_{1}$ and $\beta_{2}$.

The particular cases of Dirichlet-Dirichlet, NeumannNeumann and Dirichlet-Neumann BC can be reobtained if we take, respectively, $\beta_{1}=\beta_{2}=0, \beta_{1}=\beta_{2} \rightarrow \infty$ and $\beta_{1}=$ $0 ; \beta_{2} \rightarrow \infty$. For the first two cases, we obtain

$$
\mathcal{F}^{D D}(a)=\mathcal{F}^{N N}(a)=-\frac{1}{32 \pi^{2} a^{4}} \int_{0}^{\infty} d \xi \frac{\xi^{3}}{e^{\xi}-1} .
$$


Using the integral representation $\int_{0}^{\infty} d \xi \frac{\xi^{s-1}}{e^{\xi}-1}=\zeta_{R}(s) \Gamma(s)$, where $\zeta_{R}$ is the Riemann zeta function, we get half the electromagnetic result, namely,

$$
\mathcal{F}^{D D}(a)=\mathcal{F}^{N N}(a)=-\frac{\pi^{2} \hbar c}{480} \frac{1}{a^{4}} .
$$

For the case of mixed $\mathrm{BC}$, we get

$$
\mathcal{F}^{D N}(a)=+\frac{1}{32 \pi^{2} a^{4}} \int_{0}^{\infty} d \xi \frac{\xi^{3}}{e^{\xi}+1}
$$

Using in the previous equation the integral representation $\int_{0}^{\infty} d \xi \frac{\xi^{s-1}}{e^{\xi}+1}=\left(1-2^{1-s}\right) \Gamma(s) \zeta_{R}(s)$ we get a repulsive pressure (equal to half of Boyer's result [60] obtained for the electromagnetic field constrained by a perfectly conducting plate parallel to an infinitely permeable one),

$$
\mathcal{F}^{D N}(a)=\frac{7}{8} \times \frac{\pi^{2} \hbar c}{480} \frac{1}{a^{4}} .
$$

\section{The Casimir effect for massive particles}

In this subsection, we sketch briefly some results concerning massive fields, just to get some feeling about what kind of influence the mass of a field may have in the Casimir effect. Firstly, let us consider a massive scalar field submitted to Dirichlet BC in two parallel plates, as before. In this case, the allowed frequencies for the field modes are given by $\omega_{\mathbf{k}}=c\left[\kappa^{2}+\frac{n^{2} \pi^{2}}{a^{2}}+m^{2}\right]^{1 / 2}$, which lead, after we use the Casimir method explained previously, to the following result for the Casimir energy per unit area (see, for instance, Ref. [38])

$$
\frac{1}{L^{2}} \mathcal{E}_{c}(a, m)=-\frac{m^{2}}{8 \pi^{2} a} \sum_{n=1}^{\infty} \frac{1}{n^{2}} K_{2}(2 a m n),
$$

where $K_{v}$ is a modified Bessel function, $m$ is the mass of the field and $a$ is the distance between the plates, as usual. The limit of small mass, am $\ll 1$, is easily obtained and yields

$$
\frac{1}{L^{2}} \mathcal{E}_{c}(a, m) \approx-\frac{\pi^{2}}{1440 a^{3}}+\frac{m^{2}}{96 a} .
$$

As expected, the zero mass limit coincides with our previous result (29) (after the force per unit area is computed). Observing the sign of the first correction on the right hand side of last equation we conclude that for small masses the Casimir effect is weakened.

On the other hand, in the limit of large mass, $a m \gg 1$, it can be shown that

$$
\frac{1}{L^{2}} \mathcal{E}_{c}(a, m) \approx-\frac{m^{2}}{16 \pi^{2} a}\left(\frac{\pi}{m a}\right)^{1 / 2} e^{-2 m a}
$$

Note that the Casimir effect disappears for $m \rightarrow \infty$, since in this limit there are no quantum fluctuations for the field anymore. An exponential decay with $m a$ is related to the plane geometry. Other geometries may give rise to power law decays when $m a \rightarrow \infty$. However, in some cases, the behaviour of the Casimir force with ma may be quite unexpected. The Casimir force may increase with $m a$ before it decreases monotonically to zero as $m a \rightarrow \infty$ (this happens, for instance, when Robin BC are imposed on a massive scalar field at two parallel plates [61]).

In the case of massive fermionic fields, an analogous behaviour is found. However, some care must be taken when computing the Casimir energy density for fermions, regarding what kind of BC can be chosen for this field. The point is that Dirac equation is a first order equation, so that if we want non-trivial solutions, we can not impose that the field satisfies Dirichlet BC at two parallel plates, for instance. The most appropriate $\mathrm{BC}$ for fermions is borrowed from the so called MIT bag model for hadrons [62], which basically states that there is no flux of fermions through the boundary (the normal component of the fermionic current must vanish at the boundary). The Casimir energy per unit area for a massive fermionic field submitted to MIT BC at two parallel plates was first computed by Mamayev and Trunov [63] (the massless fermionic field was first computed by Johnson in 1975 [64])

$$
\begin{gathered}
\frac{1}{L^{2}} \mathcal{E}_{c}^{f}(a, m)= \\
-\frac{1}{\pi^{2} a^{3}} \int_{m a}^{\infty} d \xi \xi \sqrt{\xi^{2}-m^{2} a^{2}} \log \left[1+\frac{\xi-m a}{\xi+m a} e^{-2 \xi}\right] .
\end{gathered}
$$

The small and large mass limits are given, respectively, by

$$
\begin{aligned}
\frac{1}{L^{2}} \mathcal{E}_{c}^{f}(a, m) & \approx-\frac{7 \pi^{2}}{2880 a^{3}}+\frac{m}{24 a^{2}} ; \\
\frac{1}{L^{2}} \mathcal{E}_{c}^{f}(a, m) & \approx-\frac{3(m a)^{1 / 2}}{2^{5} \pi^{3 / 2} a^{3}} e^{-2 m a} .
\end{aligned}
$$

Since the first correction to the zero mass result has an opposite sign, also for a fermionic field small masses diminish the Casimir effect. In the large mass limit, $m a \rightarrow \infty$, we have a behaviour analogous to that of the scalar field, namely, an exponential decay with $m a$ (again this happens due to the plane geometry). We finish this section with an important observation: even a particle as light as the electron has a completely negligible Casimir effect.

\section{MISCELLANY}

In this section we shall briefly present a couple of topics which are in some way connected to the Casimir effect and that have been considered by our research group in the last years. For obvious reasons, we will not be able to touch all the topics we have been interested in, so that we had to choose only a few of them. We first discuss how the Casimir effect of a charged field can be influenced by an external magnetic field. Then, we show how the constitutive equations associated to the Dirac quantum vacuum can be affected by the 
presence of material plates. Finally, we consider the so called dynamical Casimir effect.

\section{A. Casimir effect under an external magnetic field}

The Casimir effect which is observed experimentally is that associated to the photon field, which is a massless field. As we mentioned previously, even the electron field already exhibits a completely unmeasurable effect. With the purpose (and hope) of enhancing the Casimir effect of electrons and positrons we considered the influence of an external electromagnetic field on their Casimir effect. Since in this case we have charged fields, we wondered if the virtual electronpositron pairs which are continuously created and destroyed from the Dirac vacuum would respond in such a way that the corresponding Casimir energy would be greatly amplified. This problem was considered for the first time in 1998 [65] (see also Ref. [66]). The influence of an external magnetic field on the Casimir effect of a charged scalar field was considered in Ref. [67] and recently, the influence of a magnetic field on the fermionic Casimir effect was considered with the more appropriate MIT BC [68].

For simplicity, let us consider a massive fermion field under anti-periodic $\mathrm{BC}$ (in the $O Z$ direction) in the presence of a constant and uniform magnetic field in this same direction. After a lengthy but straightforward calculation, it can be shown that the Casimir energy per unit area is given by [66]

$$
\begin{aligned}
\frac{\mathcal{E}(a, B)}{\ell^{2}} & =-\frac{2(a m)^{2}}{\pi^{2} a^{3}} \sum_{n=1}^{\infty} \frac{(-1)^{n-1}}{n^{2}} K_{2}(a m n) \\
& -\frac{e B}{4 \pi^{2} a} \sum_{n=1}^{\infty}(-1)^{n-1} \int_{0}^{\infty} d \sigma e^{-(n / 2)^{2} \sigma-(a m)^{2} / \sigma} L\left(\frac{e B a^{2}}{\sigma}\right)
\end{aligned}
$$

where we introduced the Langevin function: $L(\xi)=\operatorname{coth} \xi-$ $1 / \xi$. In the strong field limit, we have

$$
\frac{\mathcal{E}(a, B)}{\ell^{2}} \approx-\frac{e B m}{\pi^{2}} \sum_{n=1}^{\infty} \frac{(-1)^{n-1}}{n} K_{1}(a m n) .
$$

In this limit we can still analyze two distinct situations, namely, the small mass limit $(m a \ll 1)$ and the large mass limit $(m a \gg 1)$. Considering distances between the plates typical of Casimir experiments ( $a \approx 1 \mu \mathrm{m})$, we have in the former case

$$
\frac{\rho_{c}(a, B)}{\rho_{c}(a, 0)} \approx 10^{-4} \times \frac{B}{\text { Tesla }} ; \quad m a \ll 1,
$$

while in the latter case,

$$
\frac{\rho_{c}(a, B)}{\rho_{c}(a, 0)} \approx 10^{-10} \times \frac{B}{\text { Tesla }} ; \quad m a \gg 1 .
$$

In the above equations $\rho_{c}(a, B)$ and $\rho_{c}(a, 0)$ are the Casimir energy density under the influence of the magnetic field and without it, respectively. Observe that for the Casimir effect of electrons and positrons, which must be treated as the large mass limit described previously, huge magnetic fields are needed in order to enhance the effect (far beyond accessible fields in the laboratory). In other words, we have shown that though the Casimir effect of a charged fermionic field can indeed be altered by an external magnetic field, the universal constants conspired in such a way that this influence turns out to be negligible and without any chance of a direct measurement at the laboratory.

\section{B. Magnetic permeability of the constrained Dirac vacuum}

In contrast to the classical vacuum, the quantum vacuum is far from being an empty space, inert and insensible to any external influence. It behaves like a macroscopic medium, in the sense that it responds to external agents, as for example electromagnetic fields or the presence of material plates. As previously discussed, recall that the (standard) Casimir effect is nothing but the energy shift of the vacuum state of the field caused by the presence of parallel plates. There are many other fascinating phenomena associated to the quantum vacuum, namely, the particle creation produced by the application of an electric field [69], the birefringence of the QED vacuum under an external magnetic field $[70,71]$ and the Scharnhorst effect $[72,73]$, to mention just a few. This last effect predicts that the velocity of light propagating perpendicularly to two perfectly conducting parallel plates which impose (by assumption) BC only on the radiation field is slightly altered by the presence of the plates. The expected relative variation in the velocity of light for typical values of possible experiments is so tiny $\left(\Delta c / c \approx 10^{-36}\right)$ that this effect has not been confirmed yet. Depending on the nature of the material plates the velocity of light propagating perpendicular to the plates is expected to diminish [74]. The Scharnhorst effect inside a cavity was considered in [75].

The negligible change in the velocity of light predicted by Scharnhorst may be connected with the fact that the effect that bears his name is a two-loop QED effect, since the classical field of a traveling light wave interacts with the radiation field only through the fermionic loop.

With the purpose of estimating a change in the constitutive equations of the quantum vacuum at the one-loop level, we were led to consider the fermionic field submitted to some BC. The magnetic permeability $\mu$ of the constrained Dirac vacuum was computed by the first time in Ref. [76], but with the nonrealistic anti-periodic $\mathrm{BC}$. In this case, the result found for the relative change in the permeability, $\Delta \mu:=\mu-1$, was also negligible (an analogous calculation has also been made in the context of scalar QED [77]). However, when the more realistic MIT BC are imposed on the Dirac field at two parallel plates things change drastically. In this case, it can be shown that the magnetic permeability of the constrained Dirac vacuum is given by [78]

$$
\frac{1}{\mu(m a)}=1-\frac{\pi-2}{12 \pi^{2}} \frac{e^{2}}{m a}+\frac{1}{6 \pi^{2}} \frac{e^{2}}{m a} H(m a),
$$


where

$$
H(m a)=\int_{1}^{\infty} d x \frac{x}{\left(x^{2}-1\right)^{3 / 2}} \ln \left\{1+\left(\frac{x-1}{x+1}\right) e^{-2 \max }\right\} .
$$

For confining distances of the order of $0,1 \mu \mathrm{m}$, we have

$$
\Delta \mu:=\mu-1 \approx 10^{-9} \text {. }
$$

The previous value is comparable to the magnetic permeability of Hydrogen and Nitrogen at room temperature and atmospheric pressure. Hence, an experimental verification of this result seems to be not unfeasible (we will come back to this point in the final remarks).

\section{The Dynamical Casimir effect}

As our last topic, we shall briefly discuss the so called dynamical Casimir effect, which consists, as the name suggests, of the consideration of a quantum field in the presence of moving boundaries. Basically, the coupling between vacuum fluctuations and a moving boundary may give rise to dissipative forces acting on the boundary as well as to a particle creation phenomenon. In some sense, these phenomena were expected. Recall that the static Casimir force is a fluctuating quantity [79] and hence, using general arguments related to the fluctuation-dissipation theorem [80] dissipative forces on moving boundaries are expected. Further, using arguments of energy conservation we are led to creation of real particles (photons, if we are considering the electromagnetic field [81]). For the above reasons, this topic is sometimes referred to as radiation reaction force on moving boundaries.

After Schwinger's suggestion that the phenomenon of sonoluminescence could be explained by the dynamical Casimir effect [82] (name coined by himself), a lot of work has been done on this subject. However, it was shown a few years later that this was not the case (see [9] and references therein for more details).

The dynamical Casimir effect already shows up in the case of one (moving) mirror [83-85]. However, oscillating cavities whose walls perform vibrations in parametric resonance with a certain unperturbed field eigenfrequency may greatly enhance the effect [86-89]. Recently, a one dimensional oscillating cavity with walls of different nature was considered [90]. The dynamical Casimir effect has also been analyzed for a variety of three-dimensional geometries, including parallel plane plates [91], cylindrical waveguides [92], and rectangular [93], cylindrical [94] and spherical cavities [95]. For a review concerning classical and quantum phenomena in cavities with moving boundaries see Dodonov [96] and for a variety of topics on non-stationary Casimir effect including perspectives of its experimental verification see the special issue [97].

In this section, we shall discuss the force exerted by the quantum fluctuations of a massless scalar field on one moving boundary as well as the particle creation phenomenon in a unusual example in 1+1 dimensions, where the field satisfies a Robin BC at the moving boundary $[98,99]$. We shall follow throughout this paper the perturbative method introduced by Ford and Vilenkin [84]. This method was also applied successfully to the case of the electromagnetic field under the influence of one moving (perfectly) conducting plate [85] as well as an oscillating cavity formed by two parallel (perfectly) conducting plates [91].

Let us then consider a massless scalar field $\phi$ in $1+1$ in the presence of one moving boundary which imposes on the field a Robin BC at one moving boundary when observed from a co-moving inertial frame. By assumption, the movement of the boundary is prescribed, non-relativistic and of small amplitude $(\delta q(t)$ is the position of the plate at a generic instant $t$ ). Last assumptions may be stated mathematically by $|\delta \dot{q}(t)|<<c$ and $|\delta q(t)|<<c / \omega_{0}$, where $\omega_{0}$ corresponds to the (main) mechanical frequency. Therefore, we must solve the following equation: $\partial^{2} \phi(x, t)=0$, with the field satisfying a Robin $\mathrm{BC}$ at the moving boundary given by

$$
\left.\left[\frac{\partial}{\partial x}+\delta \dot{q}(t) \frac{\partial}{\partial t}\right] \phi(x, t)\right|_{x=\delta q(t)}=\left.\frac{1}{\beta} \phi(x, t)\right|_{x=\delta q(t)} .
$$

where $\beta$ is a non-negative parameter and the previous condition was already written in the laboratory frame. We are neglecting terms of the order $O\left(\delta \dot{q}^{2} / c^{2}\right)$. The particular cases of Dirichlet and Neumann BC are reobtained by making $\beta=0$ and $\beta \rightarrow \infty$, respectively. The dissipative forces for these particular cases were studied at zero temperature as well as at finite temperature and also with the field in a coherent state in [100] (dissipative forces on a perfectly conducting moving plate caused by the vacuum fluctuations of the electromagnetic field at zero and non-zero temperature were studied in [101]). The perturbative approach introduced by Ford and Vilenkin [84] consists in writing

$$
\phi(x, t)=\phi_{0}(x, t)+\delta \phi(x, t),
$$

where $\phi_{0}(x, t)$ is the field submitted to a Robin BC at a static boundary fixed at the origin and $\delta \phi(x, t)$ is the first order contribution due to the movement of the boundary. The total force on the moving boundary may be computed with the aid of the corresponding energy-momentum tensor, namely,

$$
\delta F(t)=\left\langle 0\left|T^{11}\left(t, \delta q^{+}(t)\right)-T^{11}\left(t, \delta q^{-}(t)\right)\right| 0\right\rangle,
$$

where superscripts + and - mean that we must compute the energy-momentum tensor on both sides of the moving boundary. It is convenient to work with time Fourier transforms. The susceptibility $\chi(\omega)$ is defined in the Fourier space by

$$
\delta \mathcal{F}(\omega)=: \chi(\omega) \delta Q(\omega)
$$

where $\delta \mathcal{F}(\omega)$ and $\delta Q(\omega)$ are the Fourier transformations of $\delta F(t)$ and $\delta q(t)$, respectively. It is illuminating to compute the total work done by the vacuum fluctuations on the moving boundary. It is straightforward to show that

$$
\int_{-\infty}^{+\infty} \delta F(t) \delta \dot{q}(t) d t=-\frac{1}{\pi} \int_{0}^{\infty} d \omega \omega \operatorname{Im} \chi(\omega)|\delta Q(\omega)|^{2} .
$$

Note that only the imaginary part of $\chi(\omega)$ appears in the previous equation. It is responsible for the dissipative effects and 
hence it is closely related with the total energy converted into real particles. On the other hand, the real part of $\chi(\omega)$, when it exists, does not contribute to the total work and hence it is not related to particle creation, but to dispersive effects. For the particular cases of Dirichlet or Neumann BC it can be shown that the susceptibility is purely imaginary and given by

$$
\chi^{D}(\omega)=\chi^{N}(\omega)=i \frac{\hbar \omega^{3}}{6 \pi c^{2}}
$$

which implies

$$
\delta F(t)=\frac{\hbar}{6 \pi c^{2}} \frac{d^{3}}{d t^{3}} \delta q(t)
$$

Since $\operatorname{Im} \chi(\omega)>0$, for these cases the vacuum fluctuations are always dissipating energy from the moving boundary.

However, for Robin $\mathrm{BC}$ an interesting thing happens. It can be shown that $\chi(\omega)$ acquires also a real part, which gives rise to a dispersive force acting on the moving boundary. The explicit expressions of $\operatorname{Re} \chi(\omega)$ and $\operatorname{Im} \chi(\omega)$ can be found in [98], but the general behaviour of them as functions of $\omega$ is shown in Figure 3. For convenience, we normalize these quantities dividing them by the value $\operatorname{Im} \chi_{D}(\omega)$, where the subscript $D$ means that $\operatorname{Im} \chi(\omega)$ must be computed with Dirichlet BC.

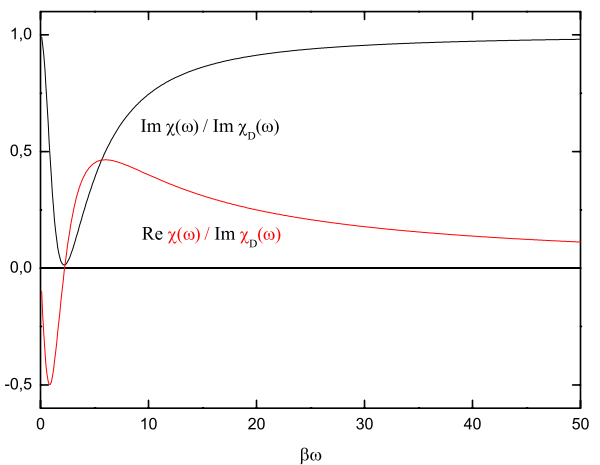

FIG. 3: Imaginary and real parts of $\chi(\omega)$ with Robin BC appropriately normalized by the value of $\operatorname{Im} \chi(\omega)$ for the Dirichlet BC.

Now, let us discuss briefly the particle creation phenomenon under Robin BC. Here, we shall consider a semi-infinite slab extending from $-\infty$ to $\delta q(t)$ following as before a prescribed non-relativistic motion which imposes on the field Robin BC at $\delta q(t)$. It can be shown that the corresponding spectral distribution is given by [99]

$$
\frac{d N}{d \omega}(\omega)=\frac{4 \omega}{1+\beta^{2} \omega^{2}} \int_{0}^{\infty} \frac{d \omega^{\prime}}{2 \pi} \frac{\left[\delta Q\left(\omega-\omega^{\prime}\right)\right]^{2}}{1+\beta^{2} \omega^{\prime 2}} \omega^{\prime}\left[1-\beta^{2} \omega \omega^{\prime}\right]^{2}
$$

As an explicit example, let us consider the particular motion

$$
\delta q(t)=\delta q_{0} e^{-|t| / T} \cos \left(\omega_{0} t\right),
$$

where, by assumption, $\omega_{0} T \gg 1$ (this is made in order to single out the effect of a given Fourier component of the motion). For this case, we obtain the following spectral distribution

$$
\begin{aligned}
\frac{d N}{d \omega}(\omega) & =\left(\delta q_{0}\right)^{2} T \omega\left(\omega_{0}-\omega\right) \times \\
& \times \frac{\left[1-\beta^{2} \omega\left(\omega_{0}-\omega\right)\right]^{2}}{\left(1+\beta^{2} \omega^{2}\right)\left(1+\beta^{2}\left(\omega_{0}-\omega\right)^{2}\right)} \Theta\left(\omega_{0}-\omega\right) .
\end{aligned}
$$

The spectral distributions for the particular cases of Dirichlet or Neumann BC can be easily reobtained by making simply $\beta=0$ and $\beta \rightarrow \infty$, respectively. The results coincide and are given by [102] (we are making $c=1$ )

$$
\frac{d N^{(D)}}{d \omega}(\omega)=\frac{d N^{(N)}}{d \omega}(\omega)=\left(\delta q_{0}\right)^{2} T \omega\left(\omega_{0}-\omega\right) \Theta\left(\omega_{0}-\omega\right) .
$$

A simple inspection in (51) shows that, due to the presence of the Heaviside step function, only the field modes with eigenfrequencies smaller than the mechanical frequency are excited. Further, the number of particles created per unit frequency when Robin BC are used is always smaller than the number of particles created per unit frequency when Dirichlet (or Neumann) BC are employed.

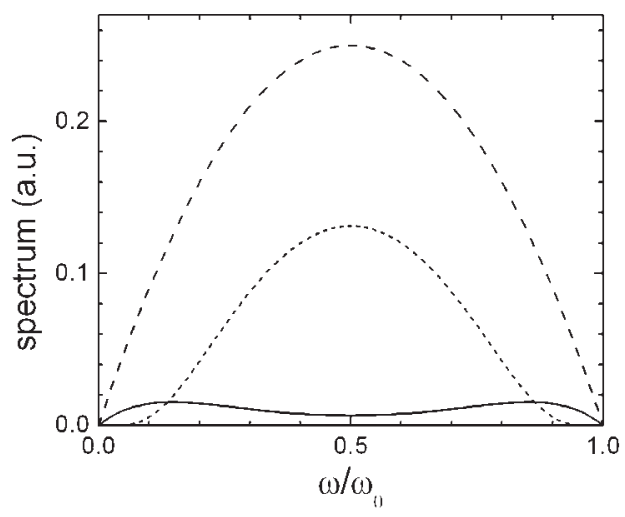

FIG. 4: Spectral distributions of created particles for: Dirichlet and Neumann BC (dashed line) and for some interpolating values of the parameter $\beta$ (dotted and solid lines).

Figure 4 shows the spectral distribution for different values of the parameter $\beta$, including $\beta=0$ (dashed line), which corresponds to Dirichlet or Neumann BC. Note that for interpolating values of $\beta$ particle creation is always smaller than for $\beta=0$ (dotted line). Depending on the value of $\beta$, particle creation can be largely suppressed (solid line).

\section{FINAL REMARKS}

In the last decades there has been a substantial increase in the study of the Casimir effect and related topics. It is remarkable that this fascinating effect, considered nowadays as a fundamental one in QFT, was born in connection with colloidal 
chemistry, an essentially experimental science. As we mentioned previously, the novelty of Casimir's seminal work [1] (see also [6]) was the technique employed by him to compute forces between neutral bodies as is emphasized by Itzykson and Zuber [103]:

\section{By considering various types of bodies influenc- ing the vacuum configuration we may give an interesting interpretation of the forces acting on them.}

However, the attractive or repulsive character of the Casimir force can not be anticipated. It depends on the specific boundary conditions, the number of space-time dimensions, the nature of the field (bosonic or fermionic), etc. The "mystery" of the Casimir effect has intrigued even proeminent physicists such as Julian Schwinger, as can be seen in his own statement [37]:

\section{... one of the least intuitive consequences of quan- tum electrodynamics.}

There is no doubt nowadays about the existence of the (static) Casimir effect, thanks to the vast list of accurate experiments that have been made during the last ten years. It is worth emphasizing that a rigorous comparison between theory and experimental data can be achieved only if the effects of temperature and more realistic BC are considered. In principle, the former are important in comparison with the vacuum contribution for large distances, while the latter can not be neglected for short distances. Typical ranges investigated in Casimir experiments are form $0.1 \mu \mathrm{m}$ to $1.0 \mu \mathrm{m}$ and, to have an idea of a typical plasma wavelengths (the plasma wavelength is closely related to the penetration depth), recall that for $\mathrm{Au}$ we have $\lambda_{P} \approx 136 \mathrm{~nm}$.

The Casimir effect has become an extremely active area of research from both theoretical and experimental points of view and its importance lies far beyond the context of QED. This is due to its interdisciplinary character, which makes this effect find applications in quantum field theory (bag model, for instance), cavity QED, atomic and molecular physics, mathematical methods in QFT (development of new regularization and renormalization schemes), fixing new constraints in hypothetical forces, nanotechnology (nanomachines operated by Casimir forces), condensed matter physics, gravitation and cosmology, models with compactified extra-dimensions, etc.

In this work, we considered quantum fields interacting only with classical boundaries. Besides, these interactions were described by highly idealized BC. Apart from this kind of interaction, there was no other interaction present. However, the fields in nature are interacting fields, like those in QED, etc. Hence, we could ask what are the first corrections to the Casimir effect when we consider interacting fields. In principle, they are extremely small. In fact, for the case of QED, the first radiative correction to the Casimir energy density (considering that the conducting plates impose BC only on the radiation field) was firstly computed by Bordag et al [104] and is given by $\mathcal{E}_{C}^{(1)}(a, \alpha)=\mathcal{E}_{C}^{(0)}(a) \frac{9}{32} \frac{\alpha \lambda_{c}}{a}$, where $\lambda_{c}$ is the Compton wavelength of the electron and $\mathcal{E}_{C}^{(0)}(a)$ is the zeroth order contribution to the Casimir energy density. As we see, at least for QED, radiative corrections to the Casimir effect are experimentally irrelevant. However, they might be relevant in the bag model, where for quarks $\lambda_{c} \approx a$ and also the quark propagators must be considered submitted to the bag $\mathrm{BC}$ [9]. Besides, the study of radiative corrections to the Casimir effect provide a good laboratory for testing the validity of idealized $\mathrm{BC}$ in higher order of perturbation theory.

Concerning the dynamical Casimir effect, the big challenge is to conceive an experiment which will be able to detect real photons created by moving boundaries or by an equivalent physical system that simulates rapid motion of a boundary. An ingenious proposal of an experiment has been made recently by the Padova's group [105]. There are, of course, many other interesting aspects of the dynamical Casimir effect that has been studied, as quantum decoherence [106], mass correction of the moving mirrors [107], etc. (see also the reviews [108]).

As a final comment, we would like to mention that surprising results have been obtained when a deformed quantum field theory is considered in connection with the Casimir effect. It seems that the simultaneous assumptions of deformation and boundary conditions lead to a new mechanism of creation of real particles even in a static situation [109]. Of course, the Casimir energy density is also modified by the deformation [110]. Quantum field theories with different space-time symmetries, other than those governed by the usual Poincaré algebra (as for example the $\kappa$-deformed Poincaré algebra [111]) may give rise to a modified dispersion relation, a desirable feature in some tentative models for solving recent astrophysical paradoxes.

Acknowledgments: I am indebted to B. Mintz, P.A. Maia Neto and R. Rodrigues for a careful reading of the manuscript and many helpful suggestions. I would like also to thank to all members of the Casimir group of UFRJ for enlightening discussions that we have maintained over all these years. Finally, I thank to $\mathrm{CNPq}$ for a partial financial support.
[1] H.B.G. Casimir Proc. K. Ned. Akad. Wet. 51, 793 (1948)

[2] F. London Z. Physik 63, 245 (1930).

[3] E.J.W. Vervey, J.T.G. Overbeek, and K. van Nes, J. Phys. and Colloid Chem. 51, 631 (1947).

[4] H.B.G. Casimir and D. Polder, Phys. Rev. 73, 360 (1948).
[5] D. Tabor and R.H.S. Winterton, Nature 219, 1120 (1968); Proc. Roy. soc. Lond. A312, 435 (1969).

[6] H.B.G. Casimir J. Chim. Phys. 46, 407 (1949).

[7] M. Planck, Verh. d. Deutsch. Phys. Ges. (2) 13 (1911).

[8] H.B.G. Casimir, Some remarks on the history of the so called 
Casimir effect, in the Proceedings of the Fourth Workshop on Quantum Field Theory under the Influence of External Conditions, pg 3, Ed. M. Bordag, World Scientific (1999).

[9] K.A. Milton, Physical Manifestation of zero point energy - The Casimir effect, World Scientific (2001).

[10] Lifshitz E M 1956 Sov. Phys. JETP 273

E.M. Lifshtz e L.P. Pitaevskii, Landau and Lifshtz Course of Theoretical Physics: Statistical Physics Part 2, ButterworthHeinemann (1980).

[11] E.S. Sabisky and C.H. Anderson, Phys. Rev. A 7, 790 (1973).

[12] Dzyaloshinskii I E Lifshitz, and L.P. Pitaevskii, Advan. Phys. 10, 165 (1961)

[13] M.J. Sparnaay, Physica 24, 751 (1958).

[14] S.K. Lamoreaux, Phys. Rev. Lett. 78, 5 (1997). S.K. Lamoreaux, Phys. Rev. Lett. 81, 5475(E) (1998).

[15] J. Blocki, J. Randrup, W.J. Swiatecki, and C.F. Tsang, Ann. of Phys. 105427 (1977).

[16] U. Mohideen e A. Roy, Phys. Rev. Lett. 81, 4549 (1998).

[17] G.L. Klimchiskaya, A. Roy, U. Mohideen, and V.M. Mostepanenko Phys. Rev. A 60, 3487 (1999).

[18] A. Roy and U. Mohideen, Phys. Rev. Lett. 82, 4380 (1999) .

[19] A. Roy, C.-Y. Lin e U. Mohideen, Phys. Rev. D 60, 111101(R) 1999

[20] U. Mohideen e A. Roy, Phys. Rev. Lett. 83, 3341 (1999).

[21] B.W. Harris, F. Chen e U. Mohideen, Phys. Rev. A 62, 052109 (2000).

[22] F. Chen, U. Mohideen, G.L. Klimchitskaya, and V.M. Mostepanenko, Phys. Rev. A 69, 022117 (2002).

[23] T. Ederth, Phys. Rev. A 62, 062104 (2000) .

[24] G. Bressi, G. Carugno, R. Onofrio e G. Ruoso, Phys. Rev. Lett. 88, 041804 (2002).

[25] F. Chen, U. Mohideen, G.L. Klimchitskaya, and V.M. Mostepanenko, Phys. Rev. Lett. 88, 101801 (2002).

F. Chen, U. Mohideen, G.L. Klimchitskaya, and V.M. Mostepanenko, Phys. Rev. A 66, 032113 (2002).

[26] R.S. Decca, E. Fischbach, G.L. Klimchitskaya, D.E. Krause, D. Lopes e V. M. Mostepanenko Phys. Rev. D 68, 116003 (2003).

[27] R.S. Decca, D. Lopez, E. Fischbach, G.L. Klimchitskaya, D.E. Krause, and V.M. Mostepanenko, Annals Phys. (NY) 318, 37 (2005).

[28] G.L.Klimchitskaya, F.Chen, R.S.Decca, E.Fischbach, D.E. Krause, D.Lopez, U.Mohideen, and V.M.Mostepanenko, J. Phys. A 39, 6485 (2006).

[29] V.M.Mostepanenko, V.B.Bezerra, R.S.Decca, B.Geyer, E.Fischbach, G.L.Klimchitskaya, D.E.Krause, D.Lopez, and C.Romero, J.Phys. A 39, 6589 (2006).

[30] A. Lambrecht and S. Reynaud, Eur. Phys. J. D 8, 309 (2000).

[31] G.L. Klimchitskaya, A. Roy, U. Mohideen, and V.M. Mostepanenko, Phys. Rev. A 60, 3487 (1999).

[32] P.A. Maia Neto, A. Lambrecht, and S. Reynaud, Phys. Rev. A 72, 012115 (2005).

[33] T.H. Boyer, Phys. Rev. 174, 1764 (1968).

[34] H.B.G. Casimir, Physica 19, 846 (1953).

[35] B. Davies, J. Math. Phys. 13, 1324 (1972)

[36] R. Balian and B. Duplantier, Ann. Phys. (N.Y.) 112, 165 (1978).

[37] K.A. Milton, L.L. DeRaad, , Jr., and J. Schwinger, Ann. Phys. (N.Y.) 115, 1 (1978).

[38] G. Plunien, B. Muller, and W. Greiner Phys. Rep. 134, 89 (1986).

[39] P.W. Milonni, The Quantum Vacuum: An Introduction to Quantum Electrodynamics, Academic, New York, (1994).

[40] V.M. Mostepanenko and N.N. Trunov, The Casimir Effect and its Applications, Clarendon Press, Oxford (1997).

[41] M. Bordag, U. Mohideen, e V. M. Mostepanenko, Phys. Rep. 353, 1 (2001).

[42] E. Elizalde, S. D. Odintsov, A. Romeo, A. A. Bitsenko, and S. Zerbini, Zeta Regularization Techniques with Applications, (World Scientific, Singapore, 1994).

[43] J. Schwinger, Lett. Math. Phys. 24, 59 (1992).

[44] M.V. Cougo-Pinto, C. Farina, A. J. Seguí-Santonja, Lett. Math. Phys. 30, 169 (1994);

M.V. Cougo-Pinto, C. Farina, A. J. Seguí-Santonja, Lett. Math. Phys. 31, 309 (1994);

L.C. Albuquerque, C. Farina, S.J. Rabello e A.N. Vaidya, Lett. Math. Phys. 34, 373 (1995).

[45] G. Barton, Proc. R. Soc. Lond. (1970).

[46] J.D. Bondurant and S.A. Fulling, J. Phys. A: Math. Gen. 38, 1505 (2005).

[47] M. Asorey, F.S. da Rosa, and L.B. Carvalho, presented in the XXV ENFPC, Caxambú, Brasil (2004).

[48] G. Chen and J. Zhou, Vibration and Damping in Distributed Systems, ol. 1 (Boca Raton, FL:CRC), pg 15.

[49] V.M. Mostepanenko and N.N. Trunov, Sov. J. Nucl. Phys. 45, 818 (1985)

[50] D. Deutsch and P. Candelas, Phys. Rev. D 20, 3063 (1979).

[51] G. Kennedy, R. Critchley, and J.S. Dowker, Ann. Phys. (NY) 125, 346 (1980).

[52] P. Minces and V.O. Rivelles, Nucl. Phys. B 572, 651 (200)

[53] S.N. Solodukhin, Phys. Rev. D 63, 044002 (2001).

[54] A. Romeo and A.A. Saharian, J. Phys. A35, 1297 (2002).

[55] M. Bordag, H. Falomir, E.M. Santangelo, and D.V. Vassilevich, Phys. Rev. D 65, 064032 (2002).

[56] S.A. Fulling, J. Phys. A 36, 6857 (2003).

[57] J.S. Dowker math.SP/0409442 v4 (2005).

[58] L.C. de Albuquerque and R.M. Cavalcanti J. Phys. A 37, 7039 (2004).

[59] L.C. de Albuquerque, hep-th/0507019 v1 (2005).

[60] T.H. Boyer, Phys. Rev A 9, 2078 (1974).

[61] T.M. Britto, C. Farina, and F.P. Reis, in preparation.

[62] A. Chodos, R.L. Jaffe, K. Johnson, C.B. Thorn, and V.F. Weisskopf, Phys. Rev. D 9, 3471 (1974).

[63] S.G. Mamayev and N.N. Trunov, Sov. Phys. J. 23, 551 (1980).

[64] K. Johnson, Acta Pol. B 6, 865 (1975).

[65] M.V. Cougo-Pinto, C. Farina, and A.C. Tort, Proceedings of the IV Workshop on Quantum Field Theory under the Influence of External Conditions, Ed. M. Bordag, Leipzig, Germany, 1998.

[66] M.V. Cougo-Pinto, C. Farina, and A.C. Tort, Braz. J. Phys. 31, 84 (2001).

[67] M.V. Cougo-Pinto, C. Farina, M.R. Negrão, and A. Tort, J. Phys. A 32, 4457 (1999).

[68] E. Elizalde, F.C. Santos, and A.C. Tort, J. Phys. A 35, 7403 (2002).

[69] J. Schwinger, Phys. Rev. 82, 664 (1951).

[70] Z. Bialynicki-Birula and I. Bialynicki-Birula, Phys. Rev. D2, 2341 (1970)

[71] S.L. Adler, Ann. Phys. (NY) 67, 599 (1971).

[72] K. Scharnhorst, Phys. Lett. B 236, 354 (1990).

[73] G. Barton, PHys. Lett. B 237, 559 (1990).

[74] M.V. Cougo-Pinto, C. Farina, F.C. Santos, and A.C. Tort, Phys. Lett. B (1998).

[75] R.B. Rodrigues and N.F. Svaiter, Physica A 342, 529 (2004).

[76] M.V. Cougo-Pinto, C. Farina, A.C. Tort, and J. Rafelski, Phys. Lett. B434, 388 (1998).

[77] M.V. Cougo-Pinto, C. Farina, M.R. Negrão, and A.C. Tort, Phys. Lett. B483, 144 (2000). 
[78] L. Bernardino, R. Cavalcanti, M.V. Cougo-Pinto, and C. Farina, to appear in the J. Phys. A.

[79] G. Barton, J. Phys. A24, 5533 (1991); C. Eberlein, J. Phys. A 25, 3015 (1992).

[80] V.B. Braginsky and F.Ya. Khalili, Phys. Lett. A161, 197 (1991);

M.T. Jaekel and S. Reynaud, Quant. Opt. 4,39 (1992).

[81] G.T. Moore, Math. Phys. 112679 (1970).

[82] J. Schwinger, Proc. R. Soc. Lond. 90, 958 (1993).

[83] S.A. Fulling and P.C.W. Davies, Proc. R. Soc. London A 348, 393 (1976).

[84] L.H. Ford and A. Vilenkin, Phys. Rev. D25, 2569 (1982).

[85] P.A. Maia Neto, J. Phys. A 27, 2167 (1994);

P. A. Maia Neto and L.A.S. Machado, Phys. Rev. A 54, 3420 (1996).

[86] C.K. Law, Phys. Rev. A 49, 433 (1994).

[87] V.V. Dodonov, Phys. Lett. A 207, 126 (1995).

[88] A. Lambrecht, M.-T. Jaekel, and S. Reynaud, Phys. Rev. Lett. 77, 615 (1996).

[89] V.V. Dodonov and A.B. Klimov, Phys. Rev. A53, 2664 (1996).

[90] D.T. Alves, E.R. Granhen, and C. Farina, Phys. Rev. A, 73, 063818 (2006).

[91] D.F. Mundarain and P.A. Maia Neto, Phys. Rev. A 57, 1379 (1998).

[92] P.A. Maia Neto, J. Opt. B: Quantum and Semiclass. Opt. 7, S86 (2005).

[93] V.V. Dodonov and A.B. Klimov, Phys. Rev. A 53, 2664 (1996).

M. Crocce, D.A.R. Dalvit and F.D. Mazzitelli, Phys. Rev. A 64, 013808 (2001).

G. Schaller, R. Schützhold, G. Plunien, and G. Soff, Phys. Rev. A 66, 023812 (2002).

[94] M. Crocce, D.A.R. Dalvit, F.C. Lombardo, and F.D. Mazzitelli, J. Opt. B: Quantum and Semiclass. Opt. 7, S32 (2005).

[95] C. Eberlein, Phys. Rev. Lett. 76, 3842 (1996); F.D. Mazzitelli and X.O. Milln, Phys. Rev. A 73, 063829 (2006).

[96] V.V. Dodonov, in: M.W. Evans (Ed.), Modern Nonlinear Optics, Advances in Chem. Phys. Series 119, 309 (Wiley, New York, 2001).

[97] Special Issue on the Nostationary Casimir effect and quantum systems with moving boundaries J. Opt. B: Quantum Semiclass. Opt. $7 \mathrm{~S} 3$, (2005).

[98] B. Mintz, C. Farina, P.A. Maia Neto and R. Rodrigues, J.
Phys. A 39, 6559 (2006)

[99] B. Mintz, C. Farina, P.A. Maia Neto and R. Rodrigues, J. Phys. A 39, 11325 (2006).

[100] D.T. Alves, C. Farina and P.A. Maia Neto, J. Phys. A 36, 11333 (2003).

[101] L.A. Machado, P.A. Maia Neto and C. Farina, Phys. Rev. D 66, 105016 (2002);

P.A. Maia Neto and C. Farina, Phys. Rev. Lett., 93, 59001 (2004).

[102] A. Lambbrecht, M.T. Jaekel and S. Reynaud, Phys. Rev. Lett. 77, 615 (1996).

[103] Claude Itzykson and Jean-Bernard Zuber, Quantum Field Theory, McGraw-Hill Book Company, New York (1980).

[104] M. Bordag, D. Robaschik, and E. Wieczorek, Ann. Phys. (NY) 165, 192 (1985).

[105] C. Braggio, G. Bressi, G. Carugno, C. Del Noce, G. Galeazzi, A. Lombardi, A. Palmieri, G. Ruoso, D. Zanello, Europhys. Lett. 70 (6), 754 (2005).

[106] D. Dalvit and P.A. Maia Neto, Phys. Rev. Lett. 84, 798 (2000); P.A. Maia Neto and D. Dalvit, Phys. Rev. A 62, 042103 (2000).

[107] M.T. Jaekel and S. Reynaud, Phys. Lett. A 180, 9 (1993); G. Barton and A. Calogeracos, Ann. Phys. (NY) 238, 227 (1995);

A. Calogeracos and G. Barton, Ann. Phys. (NY) 238, 268 (1995);

M.T. Jaekel and S. Reynaud, J. Physique13, 1093 (1993); L.A.S. Machado and P.A. Maia Neto, Phys. Rev. D65, 125005 (2002).

[108] M.T. Jaekel and S. Reynaud, Rep. Prog. Phys. 60, 863 (1997); R. Golestanian and M. Kardar, Rev. Mod. Phys. 71, 1233 (1999).

[109] M.V. Cougo-Pinto and C. Farina, Phys. Lett. B 391, 67 (1997);

M.V. Cougo-Pinto, C. Farina and J.F.M. Mendes, Phys. Lett. B 529, 256 (2002).

M.V. Cougo-Pinto, C. Farina, and J.F.M. Mendes, Preprint hep-th/0305157 (2003);

[110] J.P. Bowes and P.D. Jarvis, Class. Quantum Grav. 13, 1405 (1996);

M.V. Cougo-Pinto, C. Farina, and J.F.M. Mendes, Nucl. Phys. B: Suppl. 127, 138 (2004).

[111] J. Lukierski, A. Nowicki, H. Ruegg, and V.N. Tolstoy, Phys. Lett. B 264, 331 (1991). 